\title{
Long non-coding RNA RP1-163G9.1 is downregulated in gastric adenocarcinoma and is associated with a poor prognosis
}

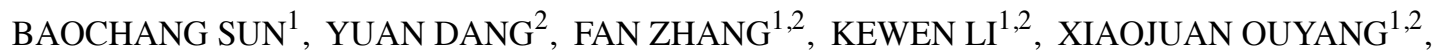 \\ $\mathrm{KAI} \mathrm{WANG}^{1,2}$ and QIAOJIA HUANG ${ }^{1,2}$ \\ ${ }^{1}$ The School of Basic Medical Sciences, Fujian Medical University, Minhou, Fuzhou, Fujian 350108; \\ ${ }^{2}$ Department of Experimental Medicine, Fuzhou General Hospital, Fuzhou, Fujian 350025, P.R. China
}

Received July 29, 2018; Accepted April 5, 2019

DOI: $10.3892 / o r .2019 .7127$

\begin{abstract}
The aim of the present study was to investigate the expression, function and underlying molecular mechanism of the long non-coding (lnc) RNA RP1-163G9.1 in patients with gastric adenocarcinoma (GA). The expression levels of IncRNA RP1-163G9.1 were determined in 112 paired clinical GA tissues by reverse transcription-quantitative polymerase chain reaction analysis. Subsequently, the potential clinical values of lncRNA RP1-163G9.1 were analyzed with statistical methods. Additionally, the function of 1ncRNA RP1-163G9.1 was explored at the cellular level using the Cell Counting Kit-8 proliferation assay, Transwell experiments, fluorescence in situ hybridization (FISH), colony formation assay and flow cytometry. Furthermore, the function of lncRNA RP1-163G9.1 was assessed in vivo using subcutaneous tumorigenesis experiments in nude mice. lncRNA RP1-163G9.1 expression in GA tissues and cells was significantly decreased when compared with that in control gastric tissues $(\mathrm{P}<0.001)$ or gastric epithelial cells GES-1 $(\mathrm{P}<0.05)$. This finding was associated with the depth of invasion $(\mathrm{P}=0.001)$, lymph node metastasis $(\mathrm{P}=0.009)$, tumor size $(\mathrm{P}=0.037)$ and immunocytochemistry marker Ki-67 $(\mathrm{P}=0.010)$. FISH detection demonstrated that lncRNA RP1-163G9.1 was primarily located in the cytoplasm. Notably, overexpression of 1ncRNA RP1-163G9.1 significantly decreased cell proliferation $(\mathrm{P}<0.01)$, colony formation $(\mathrm{P}<0.01)$, invasion $(\mathrm{P}<0.01)$ and the number of cells at the $\mathrm{S}$-phase of the cell cycle $(\mathrm{P}<0.05)$; However, it did not exert a significant effect on apoptosis $(\mathrm{P}>0.05)$. Furthermore, tumor formation experiments revealed that overexpression of lncRNA RP1-163G9.1 inhibited cancer cell proliferation in nude mice. The present research indicated that low expression
\end{abstract}

Correspondence to: Professor Qiaojia Huang or Dr Baochang Sun, The School of Basic Medical Sciences, Fujian Medical University, 1 Xueyuan Road, Minhou, Fuzhou, Fujian 350108, P.R. China

E-mail: huangqj100@126.com

E-mail: sbch1532@126.com

Key words: long non-coding RNA RP1-163G9.1, expression, function, gastric adenocarcinoma of lncRNA RP1-163G9.1 may be associated with enhanced tumor proliferation and invasion in GA.

\section{Introduction}

Gastric cancer is one of the most common types of cancer and the second most common cause of cancer-related mortality worldwide $(1,2)$. Furthermore, gastric cancer is also one of the most common malignant tumors and the second leading cause of cancer-related mortality in China. Among malignant tumors of the stomach, gastric adenocarcinoma (GA) is the most common histological subtype, accounting for $95 \%$ of total morbidity. Early-stage GA rarely causes symptoms or only causes mild symptoms. However, once clinical symptoms eventually manifest, the chances of successful treatment are reduced (3). Therefore, it is crucial to identify landmark genes or small molecules that are associated with the proliferation and metastasis of GA and may be used as diagnostic biomarkers and drug targets for GA treatment $(4,5)$.

Long non-coding (lnc) RNAs are transcripts $>200$ nucleotides in length that do not encode proteins. IncRNAs were initially considered to be byproducts of RNA polymerase II transcription (a type of genomic 'noise') and to have no physiological function $(6,7)$. However, recent studies revealed that lncRNAs can in fact regulate gene expression in different processes, including transcriptional and post-transcriptional regulation, translation and epigenetic regulation $(8,9)$. This identification complements the traditional genetic law. Notably, only $\sim 1.5 \%$ of the genome is gene-coding, and the majority of these genes are transcribed into non-coding sequences, which account for $9-11 \%$ of total RNA. According to their location relative to protein-coding genes, IncRNAs may be divided into five types, namely antisense, sense-overlapping, intronic, bidirectional and intergenic (10). The positioning of lncRNAs may help identify their function. Notably, various lncRNAs are associated with human cancer. Previous studies have indicated that the aberrant expression of lncRNAs may be associated with the occurrence and development of tumors. For example, lncRNA HOX transcript antisense RNA upregulation in GA increases gastric cancer cell progression through recruitment of microRNA (miR)-331-3p and regulation of human epidermal growth factor receptor 2 gene expression by means of competing endogenous (ce)RNA (11). In addition, inhibition 
of lncRNA H19 can suppress the growth of myeloma cells through the nuclear factor- $\kappa \mathrm{B}$ signaling pathway (12). c-Myc gene-induced $\mathrm{H} 19$ can also promote cell proliferation (13). H19 has been shown to be able to promote gastric cancer cell proliferation via miR-675 through the tumor suppressor runt domain transcription factor 1 (14). According to previous research (15), IncRNAs are stable in blood specimens and can be examined using fluorescence quantitative polymerase chain reaction (qPCR) technology. Although various cancer-associated lncRNAs have been identified, their function remains to be elucidated. Currently, it remains unclear how several lncRNAs are involved in important biological processes, including tumor growth, proliferation and differentiation.

GA is one of the most frequently occurring cancer types in the Fujian area in China. Therefore, our previous study aimed to investigate the lncRNA expression profile in tissue samples from patients with GA using a high-throughput chip assay technique (16). A number of lncRNAs were found to be differentially expressed in GA tissues compared with paired non-cancerous tissues. The results of the chip studied by our group revealed that IncRNA RP1-163G9.1 was expressed at low levels in GA tissue compared with its expression in adjacent control tissues; however, its specific biological functions and possible mechanisms of action remain unclear. To the best of our knowledge, no previous research has indicated the role of lncRNA RP1-163G9.1 in gastric cancer or other types of cancer. Therefore, the aim of the present study was to assess the role of lncRNA RP1-163G9.1 in GA.

lncRNA RP1-163G9.1 is located in chromosome 1:2976180-2978596, which contains three exons (1,697 bp in size). Based on our previous data from microarray chip assays, lncRNA RP1-163G9.1 was found to be downregulated in GA tissues. To the best of our knowledge, the association of this IncRNA with GA has not yet been reported. Therefore, it may be of value to study the function and possible underlying mechanism of lncRNA RP1-163G9.1 in GA. In the present study, the possible association of this IncRNA with GA was investigated in vitro and in vivo and its potential usefulness as a molecular diagnostic marker was also evaluated.

\section{Materials and methods}

Specimen collection and preservation. A total of 112 fresh clinically diagnosed GA specimens and their paired non-cancerous tissues (collected at a distance of $\geq 5 \mathrm{~cm}$ from the tumor) were collected between April 2014 and August 2016, and immediately placed into RNase-free cryopreserved tubes containing RNAlater solution (Thermo Fisher Scientific, Inc., Waltham, MA, USA). The specimens were numbered with a unique ID code and labeled with specific information, including storage time and tissue type. The specimens were stored in a cryogenic refrigerator at $-80^{\circ} \mathrm{C}$ for later use.

Data collection on clinicopathological parameters. Patient information, including age, sex, tumor location and size, depth of invasion, differentiation, lymph node metastasis, histological type and immunohistochemical markers, was collected following gastric cancer excision using the hospital's Laboratory Information System, pathological diagnosis system and database.
$R N A$ extraction and reverse transcription-qPCR (RT-qPCR). Total RNA was extracted from fresh GA specimens and GES-1, BGC-823, SGC7901, MGC803 and AGS cells using TRIzol reagent (Invitrogen; Thermo Fisher Scientific, Inc.). A total of $200 \mathrm{ng}$ RNA was used to synthesize cDNA with EasyScript One-Step gDNA Removal and cDNA Synthesis SuperMix (cat. no. AE311; TransGen Biotech, Co., Ltd., Beijing, China). According to the known gene sequence, specific primers of lncRNA RP1-163G9.1 and GAPDH were designed using the National Center for Biotechnology Information online primer design program Primer 3.0. All primers were synthesized by Sangon Biotech Co., Ltd. (Shanghai, China). The sequences of primers were as follows: GAPDH, forward 5'-ACCCAC TCCTCCACCTTTGAC-3' and reverse, 5'-TGTTGCTGT AGCCAAATTCGTT-3'; and IncRNA RP1-163G9.1, forward 5'-CGCCTCTCACTGGTAAGTCC-3' and reverse, 5'-AAC TGAGTCCCCAAAGACCC-3'. The relative expression of IncRNA RP1-163G9.1 was determined using RT-qPCR and SYBR-Green reagent from a TransStartR qPCR Super Mix kit (cat. no. AQ131; TransGen Biotech, Co., Ltd.). The qPCR conditions were as follows: $95^{\circ} \mathrm{C}$ for $3 \mathrm{~min}, 94^{\circ} \mathrm{C}$ for $5 \mathrm{sec}$ and $58^{\circ} \mathrm{C}$ for $30 \mathrm{sec}$ for 40 cycles, followed by a dissociation stage at $95^{\circ} \mathrm{C}$ for $15 \mathrm{sec}, 60^{\circ} \mathrm{C}$ for $15 \mathrm{sec}$ and $95^{\circ} \mathrm{C}$ for $15 \mathrm{sec}$. The $2^{-\Delta \Delta C q}$ method was used to detect the expression fold changes of 1ncRNA RP1-163G9.1. GADPH was used as the internal control. The standard curve was constructed to determine the amplification efficiency.

Fluorescence in situ hybridization (FISH). A total of 30 paired paraffin-embedded sections were selected from the 112 pairs of GA specimens to determine the expression localization of lncRNA RP1-163G9.1 using FISH. The organization paraffin block was provided by the Department of Pathology, Fuzhou General Hospital (Fuzhou, China). The probe sequence of IncRNA RP1-163G9.1 was designed and synthesized by GenePharma Co., Ltd. (Shanghai, China) and marked with 5'CY3. The sequence was as follows: 5'-CTGCCGCCACCG TTCTACC-3'. Paraffin sections were prepared by placing the paraffin block on ice for $1 \mathrm{~h}$, cutting the samples into $3-\mu \mathrm{m}$ slices and then naturally drying the slices. Hybridization was conducted on a ThermoBrite hybrid instrument (Qi Wei Industrial Co.,Ltd., Shanghai, China) overnight after sealing. The probe concentration was $5 \mu \mathrm{M}$. The hybridization conditions were as follows: $75^{\circ} \mathrm{C}$ for $5 \mathrm{~min}$ followed by incubation at $42^{\circ} \mathrm{C}$ for $16 \mathrm{~h}$.

Ethics statement. The present study was approved by the Fuzhou General Hospital Ethics Committee. All patients provided permission for the use of their tissue samples for research purposes.

Scoring criteria of FISH. The cytoplasm was stained red and identified as lncRNA RP1-163G9.1-positive expression. According to Bai et al (17), staining intensity was scored as follows: No staining [immunoreactive score (IRS): 0], weakly positive (IRS: 1-2), moderately positive (IRS: $3-6$ ), strongly positive (IRS: 8-12). All results were determined by two different members of staff at the same time.

Cell lines and culture conditions. Normal gastric epithelial cells (GES-1) were obtained from Beijing Institute of Cancer 
Prevention (Beijing, China), and the GA cell lines (BGC-823 and MGC-803) were obtained from Cell Bank, Chinese Academy of Sciences (Shanghai, China). The AGS and SGC-7901 GA cell lines were purchased from the American Type Culture Collection (Manassas, VA, USA). GES-1 cells were cultured in Dulbecco's modified Eagle's medium (DMEM) supplemented with $10 \%$ fetal bovine serum (FBS) (Gibco; Thermo Fisher Scientific, Inc.). AGS cells were cultured in F12 medium with 10\% FBS. BGC-823, MGC-803 and SGC07901 cells were cultured in RPMI-1640 supplemented with $10 \%$ FBS. The cells were cultured at $37^{\circ} \mathrm{C}$ in a humidified atmosphere containing $5 \% \mathrm{CO}_{2}$ in culture flasks. The medium was changed every 1-2 days.

Screening clones that stably express $p C D N A 3.1-P R 1$ and $p C D N A 3.1$. The vectors of pCDNA3.1-lncRNA RP1-163G9.1 (pCDNA3.1-PR1), pCDNA3.1-GFP and pCDNA3.1 were purchased from GenePharma Co., Ltd. pCDNA3.1-PR1 vector, which overexpressed lncRNA RP1-163G9.1, was constructed by sequence ligation of lncRNA RP1-163G9.1 (1,697 bp) with pcDNA3.1 empty vector via BamHI and EcoRI sites. In order to determine the most suitable concentration, G418 (Nuoyang Biological Co., Ltd., Hangzhou, China) was diluted from 300 to $1,100 \mu \mathrm{g} / \mathrm{ml}$ in nine consecutive concentrations for drug screening with BGC-823 and SGC7901 cells. BGC-823 and SGC7901 were digested with EDTA-trypsin, followed by subculturing in 6 -well plates at $1 \times 10^{5}$ cells/well. After the cells had grown to $60-70 \%$ confluence, they were transfected with pCDNA3.1-PR1, pCDNA3.1-GFP and pCDNA3.1 using Lipofectamine 2000 transfection reagent (Invitrogen; Thermo Fisher Scientific, Inc.). pcDNA3.1-GFP was used to monitor transfection efficiency and G418 was used at an appropriate concentration to determine stable clone screening following a 24-h transfection. Culture solution and G418 were changed every 3-4 days until cell clones could be observed by the naked eye. Single clones were selected and culture was continued with G418.

Cell proliferation experiment. Cells stably expressing pCDNA3.1-PR1 and empty vector pCDNA3.1 were digested in a single-cell suspension solution, seeded into 96 -well plates at a density of 2,000 cells/well and cultured at $37^{\circ} \mathrm{C}$ in an atmosphere containing $5 \% \mathrm{CO}_{2}$. A total of six replicate wells were used. According to the manufacturer's instructions, $10 \mu \mathrm{l} /$ well of Cell Counting Kit-8 (CCK-8) reagent (Nuoyang Biological Co., Ltd., Hangzhou, China) was added to the cells, followed by incubation at $37^{\circ} \mathrm{C}$ for $1 \mathrm{~h}$. Absorbance was measured at 450 and $630 \mathrm{~nm}$ using a microplate reader (Spectra Max 190; Molecular Devices, LLC, Sunnyvale, CA, USA). The assays were performed on days 0 (following culture for $6 \mathrm{~h}$ ), 1, 2, 3 and 4 , and a cell proliferation curve was drawn.

Colony formation experiments. Single-cell suspension solutions of cells stably expressing pcDNA3.1-PR1 and empty vector pcDNA3.1 were prepared and subcultured in 6-well plates at a density of 1,000 cells/well. Culturing was performed at $37^{\circ} \mathrm{C}$ in an atmosphere containing $5 \% \mathrm{CO}_{2}$ for 14 days. When a single colony contained $\geq 50$ cells, the cells were fixed with methanol for $15 \mathrm{~min}$ and stained with $0.1 \%$ crystal violet solution for $15 \mathrm{~min}$. A total of 10 fields of vision were randomly selecting per well and the number of colonies was counted under a microscope. Three independent experiments were conducted.

Detection of apoptosis and analysis of the cell cycle. BGC823 cells, which stably expressed lncRNA RP1-163G9.1 and pcDNA3.1, were digested by trypsin. A single-cell suspension containing $5 \times 10^{5}$ cells was prepared. According to the instructions of the manufacturer of Annexin V-fluorescein isothiocyanate (FITC)/propidium iodide (PI) apoptosis detection kits (Nanjing KeyGen Biotech Co., Ltd., Nanjing, China), following staining for $5 \mathrm{~min}$ at room temperature away from light with $5 \mu \mathrm{l}$ PI and $5 \mu \mathrm{l}$ Annexin V-FITC, apoptosis was detected using a flow cytometry instrument (BD FACSCalibur; Becton, Dickinson and Company, San Jose, CA, USA). A total of $5 \times 10^{5}$ cells in the logarithmic growth phase were collected, resuspended and stained with $5 \mu \mathrm{l}$ PI for $10 \mathrm{~min}$ at room temperature away from light. Subsequently, the cell cycle was analyzed using a flow cytometry instrument.

Transwell experiments. The Transwell chamber (EMD Millipore; Billerica, MA, USA) was prepared with a BD Matrigel. The mixing ratio of DMEM and BD Matrigel was 1:4. The chamber preparation experiment was completed on ice and the temperature did not exceed $10^{\circ} \mathrm{C}$. A total of $100 \mu \mathrm{l}$ cell suspension containing $5 \times 10^{4}$ cells was added to the upper Transwell chambers. A total of $600 \mu 1$ complete medium containing serum supplemented with $10 \%$ FBS was added to the lower chamber and the cells were cultured at $37^{\circ} \mathrm{C}$ in an atmosphere containing $5 \% \mathrm{CO}_{2}$. The experiments were terminated following $48 \mathrm{~h}$ of incubation. The chamber was washed three times with phosphate-buffered saline (PBS), fixed with methanol for $20 \mathrm{~min}$ and then stained with $0.1 \%$ crystal violet solution for $20 \mathrm{~min}$. Excess dye was removed with PBS and cells in the interior of the chamber were removed with a cotton swab. Five fields of view were selected, and the number of cells was counted using an inverted microscope (Olympus IX51; Olympus Corporation, Tokyo, Japan).

In vivo experiments. A total of $20 \mathrm{BALB} / \mathrm{c}$ male nude mice (aged 4-6 weeks and weighing 16-20 g) were purchased from Slac Laboratory Animal Co., Ltd. (Shanghai, China). The mice were equally divided into two groups. Single-cell suspensions using PBS and SGC7901 cells stably overexpressing pCDNA3.1 and pCDNA3.1-PR1 were prepared and subcutaneously inoculated in the right flank of nude mice $\left(8 \times 10^{6}\right.$ cells $\left./ 0.1 \mathrm{ml} / \mathrm{mouse}\right)$. Nude mice were observed every day and the tumor volume was measured every week. Tumor volume was calculated as follows: $\mathrm{V}\left(\mathrm{mm}^{3}\right)=\mathrm{ab}^{2} / 2$, where ' $\mathrm{a}$ ' is the long diameter and ' $b$ ' the short diameter of the measured tumors. After 5 weeks, the nude mice were euthanized by cervical dislocation, the tumors were removed and the volumes and weights were determined, and hematoxylin and eosin staining was performed on representative specimens.

Statistical analysis. The relative expression level of lncRNA RP1-163G9.1 was obtained by conducting statistical analysis with a paired t-test using GraphPad Prism 5.0 (GraphPad Software, Inc., La Jolla, CA, USA). All clinicopathological data were assessed using SPSS 19.0 software (IBM Corp., 

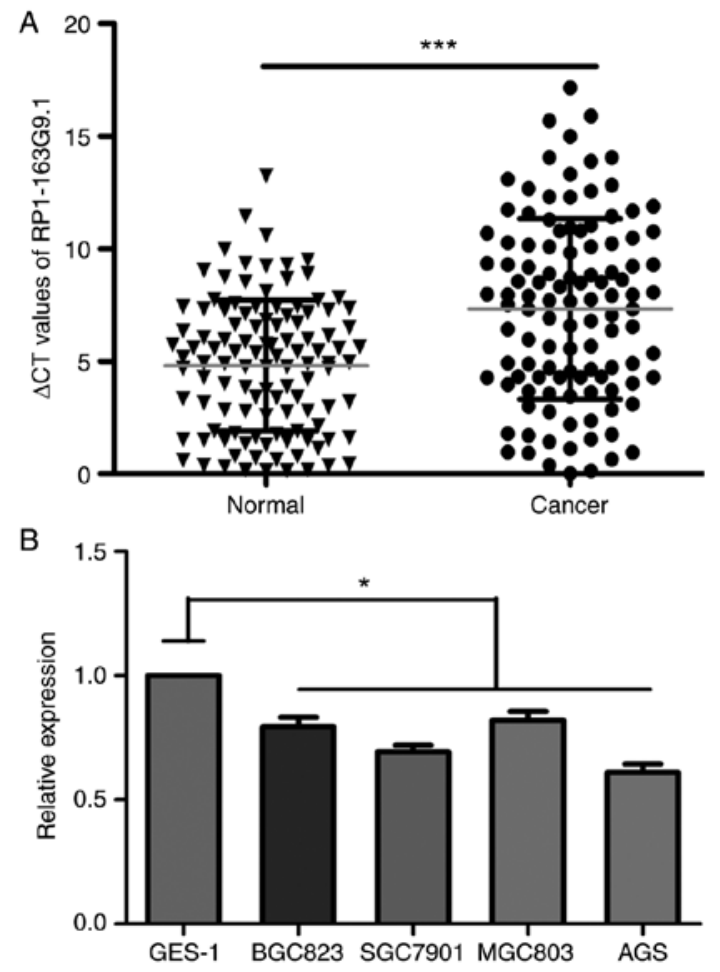

Figure 1. Expression level of lncRNA RP1-163G9.1. (A) Compared with control tissues, the expression of 1ncRNA RP1-163G9.1 in GA tissues was downregulated ( $\left.\mathrm{n}=112,{ }^{* * *} \mathrm{P}<0.001\right)$. (B) Relative expression levels of lncRNA RP1-163G9.1 in GA cells and normal gastric epithelial cells (GES-1). Data were obtained from three independent experiments and are displayed as the mean \pm standard deviation. ${ }^{*} \mathrm{P}<0.05,{ }^{* * *} \mathrm{P}<0.001$. IncRNA, long non-coding RNA; GA, gastric adenocarcinoma.

Armonk, NY, USA). The correlation of 1ncRNA RP1-163G9.1 expression with clinical pathological parameters and immunohistochemical markers was assessed using analysis of variance. Cell function experiments were conducted by a double-sided t-test and $\mathrm{P}<0.05$ was considered to indicate a statistically significant difference. Data are presented as the mean \pm standard deviation calculated from three replicate representative experimental results. In addition, the association of lncRNA RP1-163G9.1 expression with the tumor-node-metastasis (TNM) stage of the tumor, lymph node metastasis and tumor size was performed using multi-factor logistic regression. $\mathrm{P}<0.05$ was considered to indicate a statistically significant difference.

\section{Results}

Expression levels of lncRNA RP1-163G9.1 in GA and adjacent non-GA tissues. IncRNA RP1-163G9.1 was found to be downregulated in GA tissues according to the results of the RT-qPCR analysis. Notably, the Cq difference between the target lncRNA RP1-163G9.1 and the internal reference gene in cancer tissues was as follows: $\Delta \mathrm{Cq}=7.317 \pm 0.379$. However, the $\mathrm{Cq}$ difference between paracancerous tissues and the internal reference gene was as follows: $\Delta \mathrm{Cq}=4.807 \pm 0.276$. Statistical analysis indicated that the expression of lncRNA RP1-163G9.1 in GA was significantly decreased compared with that in adjacent non-GA tissues $(\mathrm{P}<0.001$; Fig. 1A). The results of tissue validation demonstrated that lncRNA
Table I. Analysis of the correlation between IncRNA RP1-163G9.1 expression level and clinicopathological parameters.

\begin{tabular}{|c|c|c|c|}
\hline $\begin{array}{l}\text { Clinicopathological } \\
\text { parameters }\end{array}$ & Cases, $\mathrm{n}$ & $\Delta \Delta \mathrm{Cq}^{\mathrm{a}}$ & P-value \\
\hline Age (years) & & & 0.643 \\
\hline$<60$ & 56 & $2.73 \pm 0.44$ & \\
\hline$\geq 60$ & 56 & $2.46 \pm 0.39$ & \\
\hline Sex & & & 0.952 \\
\hline Male & 80 & $2.59 \pm 0.35$ & \\
\hline Female & 32 & $2.62 \pm 0.52$ & \\
\hline Differentiation & & & 0.074 \\
\hline Poor & 63 & $3.10 \pm 0.38$ & \\
\hline Moderate & 46 & $1.85 \pm 0.46$ & \\
\hline High & 3 & $4.36 \pm 1.52$ & \\
\hline Depth of invasion & & & 0.001 \\
\hline $\mathrm{T} 1+\mathrm{T} 2$ & 23 & $0.39 \pm 0.56$ & \\
\hline $\mathrm{T} 3+\mathrm{T} 4$ & 89 & $3.17 \pm 0.31$ & \\
\hline Lymph node metastasis & & & 0.009 \\
\hline No & 35 & $1.49 \pm 0.58$ & \\
\hline Yes & 77 & $3.11 \pm 0.32$ & \\
\hline Tumor size, $\mathrm{cm}$ & & & 0.037 \\
\hline$<4$ & 50 & $1.93 \pm 0.44$ & \\
\hline$\geq 4$ & 62 & $3.15 \pm 0.37$ & \\
\hline Neural invasion & & & 0.946 \\
\hline No & 62 & $2.62 \pm 0.42$ & \\
\hline Yes & 50 & $2.58 \pm 0.39$ & \\
\hline Vessel invasion & & & 0.291 \\
\hline No & 58 & $2.90 \pm 0.43$ & \\
\hline Yes & 54 & $2.28 \pm 0.38$ & \\
\hline
\end{tabular}

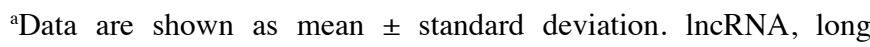
non-coding RNA.

RP1-163G9.1 expression was consistent with the data of the chip analysis. The RT-qPCR results revealed that IncRNA RP1-163G9.1 expression was downregulated in 85/112 cases of GA $(75.89 \%)$ by 23.94 -fold. Notably, the expression of lncRNA RP1-163G9.1 was also detected in gastric cancer cell lines (BGC823, SGC7901, MGC803 and AGS) and the control gastric epithelial cells (GES-1). 1ncRNA RP1-163G9.1 expression was also significantly downregulated in the four gastric cancer cells compared with GES-1 cells ( $\mathrm{P}<0.05$; Fig. 1B).

Correlation analysis of lncRNA RP1-163G9.1 expression with clinicopathological parameters and immunohistochemical markers. Patients' detailed clinical data were collected and correlation analysis of 1ncRNA RP1-163G9.1 expression with clinicopathological parameters was performed. The results suggested that decreased expression of lncRNA RP1-163G9.1 was significantly associated with the depth of tumor invasion $(\mathrm{P}=0.001)$, lymph node metastasis $(\mathrm{P}=0.009)$ and tumor size $(\mathrm{P}=0.037)$ (Table I). These findings suggested 
Table II. Correlation analysis between lncRNA RP1-163G9.1 expression level and immunohistochemical markers.

\begin{tabular}{|c|c|c|c|}
\hline Immunohistochemical markers & Cases, $\mathrm{n}$ & $\Delta \Delta \mathrm{Cq}^{\mathrm{a}}$ & P-value \\
\hline$\alpha$-fetoprotein & & & 0.607 \\
\hline Low & 102 & $2.55 \pm 0.31$ & \\
\hline High & 10 & $3.08 \pm 0.65$ & \\
\hline Carcinoembryonic antigen & & & 0.113 \\
\hline Low & 93 & $2.39 \pm 0.32$ & \\
\hline High & 19 & $3.62 \pm 0.72$ & \\
\hline Carbohydrate antigen $19-9$ & & & 0.287 \\
\hline Low & 94 & $2.47 \pm 0.32$ & \\
\hline High & 18 & $3.31 \pm 0.30$ & \\
\hline Vascular endothelial growth factor & & & 0.715 \\
\hline Low & 34 & $2.76 \pm 0.49$ & \\
\hline High & 78 & $2.53 \pm 0.36$ & \\
\hline C-erbB-2 & & & 0.932 \\
\hline Low & 98 & $2.61 \pm 0.31$ & \\
\hline High & 14 & $2.54 \pm 0.91$ & \\
\hline Thymidine synthase & & & 0.076 \\
\hline Low & 53 & $2.06 \pm 0.42$ & \\
\hline High & 59 & $3.09 \pm 0.40$ & \\
\hline Breast cancer type 1 & & & 0.859 \\
\hline Low & 32 & $2.52 \pm 0.48$ & \\
\hline High & 80 & $2.63 \pm 0.36$ & \\
\hline Excision repair cross-complementation group 1 & & & 0.199 \\
\hline Low & 31 & $3.21 \pm 0.60$ & \\
\hline High & 81 & $2.37 \pm 0.33$ & \\
\hline Protamine 1 & & & 0.566 \\
\hline Low & 77 & $2.49 \pm 0.35$ & \\
\hline High & 35 & $2.85 \pm 0.52$ & \\
\hline $\mathrm{Ki}-67$ & & & 0.010 \\
\hline Low & 37 & $1.54 \pm 0.48$ & \\
\hline High & 75 & $3.20 \pm 0.35$ & \\
\hline
\end{tabular}

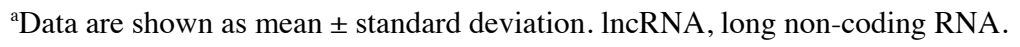

that low lncRNA RP1-163G9.1 expression may promote tumor cell proliferation and invasion. The correlation of the decreased expression of lncRNA RP1-163G9.1 with immunohistochemical markers of GA was also assessed. The results demonstrated that decreased lncRNA RP1-163G9.1 expression was significantly associated with Ki-67 ( $\mathrm{P}=0.010$; Table II). $\mathrm{Ki}-67$ is a known cell proliferation marker. Notably, a higher $\mathrm{Ki}-67$-positive rate is indicative of faster tumor proliferation and a higher degree of malignancy. Therefore, our results suggested that 1ncRNA RP1-163G9.1 may be associated with tumor cell proliferation. Multivariate logistic regression analysis also indicated that lncRNA RP1-163G9.1 acted as a tumor-protective factor $(\mathrm{P}<0.05)$. The adjusted variables were age, sex, tumor differentiation and histological type (Table III).

In situ expression and localization of IncRNA RP1-163G9.1. A total of 30 pairs of GA paraffin-embedded tissues were selected to conduct FISH. The results demonstrated that lncRNA RP1-163G9.1 was primarily expressed in the cytoplasm, whereas no expression was detected in the nucleus (Fig. 2A). Compared with paired control tissues, the expression of lncRNA RP1-163G9.1 was significantly lower compared with that of their paired control tissues when analyzed with GraphPad Prism 5 software (P<0.001; Fig. 2B).

Screening of clones stably expressing lncRNA RP1-163G9.1 in BGC-823 and SGC-7901 cells. G418 drug screenings of BGC823 and SGC7901 cells were completed and the final concentration of G418 was 800 and $700 \mu \mathrm{g} / \mathrm{ml}$, respectively. SGC7901 and BGC823 clones exhibiting stable overexpression of 1ncRNA RP1-163G9.1 were successfully constructed. The relative expression of lncRNA RP1-163G9.1 was detected with RT-qPCR. Notably, the expression of 1ncRNA RP1-163G9.1 in the pCDNA3.1-PR1 group was significantly increased 
Table III. Logistic regression analysis of lncRNA RP1-163G9.1 and tumor depth of invasion, LMN and tumor size.

\begin{tabular}{|c|c|c|c|c|c|c|c|}
\hline \multirow{2}{*}{$\begin{array}{l}\text { lncRNA RP1-163G9.1 } \\
\text { expression }\end{array}$} & \multirow{2}{*}{$\begin{array}{l}\text { LNM (n) } \\
\text { No/yes }\end{array}$} & \multicolumn{3}{|c|}{ Unadjusted } & \multicolumn{3}{|c|}{ Adjusted $^{\mathrm{a}}$} \\
\hline & & OR & $\mathrm{CI}$ & P-value & OR & $\mathrm{CI}$ & P-value \\
\hline Low & $20 / 65$ & \multicolumn{3}{|c|}{ Reference } & \multicolumn{3}{|c|}{ Reference } \\
\hline High & $15 / 12$ & 0.25 & $0.10-0.61$ & 0.001 & 0.21 & $0.08-0.57$ & 0.002 \\
\hline \multirow{2}{*}{$\begin{array}{l}\text { lncRNA RP1-163G9.1 } \\
\text { expression }\end{array}$} & \multirow{2}{*}{$\begin{array}{l}\text { Invasion depth } \\
\mathrm{T} 1+\mathrm{T} 2 / \mathrm{T} 3+\mathrm{T} 4(\mathrm{n})\end{array}$} & \multicolumn{3}{|c|}{ Unadjusted } & \multicolumn{3}{|c|}{ Adjusted $^{\mathrm{a}}$} \\
\hline & & OR & $\mathrm{CI}$ & P-value & OR & $\mathrm{CI}$ & P-value \\
\hline Low & $10 / 75$ & \multicolumn{3}{|c|}{ Reference } & \multicolumn{3}{|c|}{ Reference } \\
\hline High & $13 / 14$ & 0.14 & $0.05-0.39$ & 0.001 & 0.14 & $0.04-0.36$ & 0.001 \\
\hline \multirow{2}{*}{$\begin{array}{l}\text { IncRNA RP1-163G9.1 } \\
\text { expression }\end{array}$} & \multirow{2}{*}{$\begin{array}{l}\text { Tumor size, cm } \\
\quad<4 / \geq 4(n)\end{array}$} & \multicolumn{3}{|c|}{ Unadjusted } & \multicolumn{3}{|c|}{ Adjusted $^{\mathrm{a}}$} \\
\hline & & OR & $\mathrm{CI}$ & P-value & OR & $\mathrm{CI}$ & $\mathrm{P}$-value \\
\hline Low & $33 / 52$ & \multicolumn{3}{|c|}{ Reference } & \multicolumn{3}{|c|}{ Reference } \\
\hline High & $17 / 10$ & 0.37 & $0.15-0.91$ & 0.028 & 0.38 & $0.15-0.93$ & 0.035 \\
\hline
\end{tabular}

${ }^{a}$ The adjusted variables were age, sex, tumor differentiation and histological type. Bold print indicates statistical significance. LMN, lymph node metastasis; lncRNA, long non-coding RNA; OR, odds ratio; CI, confidence interval.

A
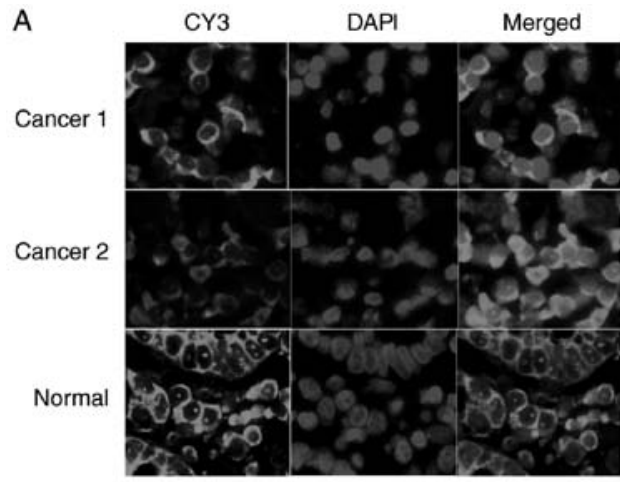

B

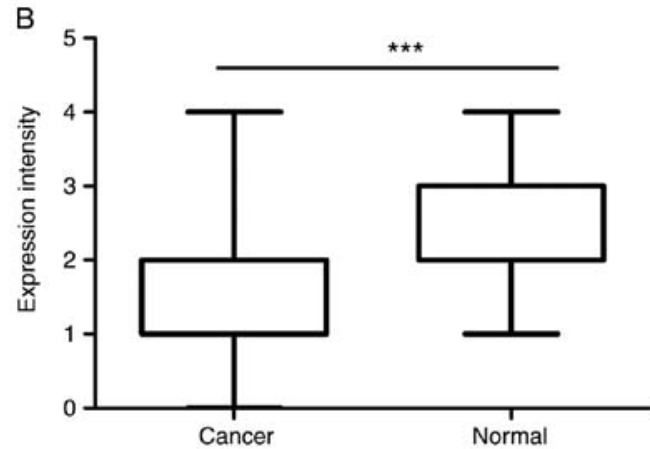

Figure 2. In situ expression and cell localization of lncRNA RP1-163G9.1 in GA and paired normal gastric tissues. 'Cancer1' and 'Cancer2' indicate the two representative gastric adenocarcinoma tissues. 'Normal' indicates the paired normal gastric tissue. (A) CY3 represents the expression of the target lncRNA RP1-163G9.1 (emitted red light under green light excitation); DAPI represents nuclear staining (emitted blue light under green light excitation); 'merge' represents the combination of the two. (B) Compared with paired normal gastric tissue, the expression of lncRNA RP1-163G9.1 was decreased in GA tissues $\left(n=30,{ }^{* * * *} \mathrm{P}<0.001\right)$. Data are presented as the mean \pm standard deviation. lncRNA, long non-coding RNA; GA, gastric adenocarcinoma; DAPI, 4',6-diamidino-2-phenylindole. compared with the pCDNA3.1 empty vector stable expression groups in SGC7901 and BGC823 cells $(\mathrm{P}<0.01)$.

Overexpression of IncRNA RP1-163G9.1 inhibits tumor cell proliferation. CCK-8 proliferation experiments were performed in BGC823 and SGC7901 cells overexpressing lncRNA RP1-163G9.1. The results indicated that the proliferation ability of the pCDNA3.1-PR1 clones was significantly lower compared with that of the pCDNA3.1 empty vector clones, indicating that lncRNA RP1-163G9.1 can inhibit the proliferation of GA cells at the cellular level ( $\mathrm{P}<0.01$; Fig. 3$)$.

Overexpression of IncRNA RPI-163G9.1 inhibits the colony-forming ability of GA cells. Colony formation experiments were performed to detect the ability of cells to form colonies. The colony-forming ability of BGC823 cells was found to be decreased in pCDNA3.1-PR1 clones compared with the empty vector control (Fig. 4A and B). Statistical analysis indicated that overexpression of lncRNA RP1-163G9.1 significantly inhibited the proliferation of tumor cells $(\mathrm{P}<0.01$; Fig. 4C).

IncRNA RP1-163G9.1 overexpression inhibits tumor cell invasion. In order to determine whether lncRNA RP1-163G9.1 affects the invasive ability of tumor cells, IncRNA RP1-163G9.1 stably overexpressing cell lines were used in Transwell experiments (Fig. 5A and B). The invasive ability of pCDNA3.1-PR1 was significantly decreased compared with the pCDNA3.1 empty vector control group in BGC-823 and SGC-7901 cells $(\mathrm{P}<0.01$; Fig. $5 \mathrm{C}$ and $\mathrm{D})$. These findings indicated that lncRNA RP1-163G9.1 may play a role in tumor cell invasion and that it may exert a tumor-protective effect. 

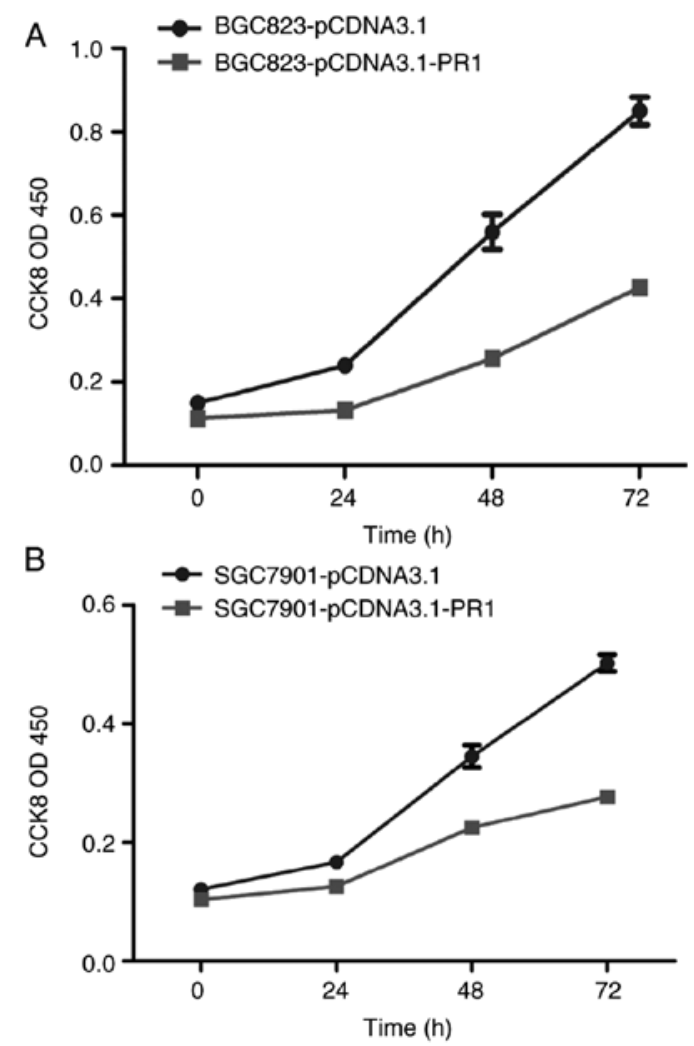

Figure 3. Cell Counting Kit-8 proliferation assay. (A and B) Proliferation curve of stable expression strains of BGC823 and SGC7901 cells. Data were obtained from three independent experiments and are presented as the mean \pm standard deviation. The results demonstrated that the proliferation in the pCDNA3.1-PR1 group was decreased compared with the pCDNA3.1 group $(\mathrm{P}<0.01)$.

Effect of IncRNA RP1-163G9.1 on the cell cycle and apoptosis of tumor cells. Apoptosis was detected using flow cytometry. The results are presented in Fig. 6A. The apoptotic level of BGC823-pCDNA3.1-PR1 did not differ significantly from that of BGC823-pCDNA3.1 ( $\mathrm{P}>0.05$; Fig. 6B). This finding indicated that lncRNA RP1-163G9.1 did not affect the apoptosis of tumor cells. Notably, cell cycle analysis demonstrated that the proportion of cells in the S-phase was significantly reduced in the BGC823-pCDNA3.1-PR1 group compared with the BGC823-pCDNA3.1 group (P<0.05; Fig. 6C and D).

Tumor growth curve. Tumor cells were subcutaneously inoculated into nude mice, and the animals were sacrificed at the end of the experiment. Tumor growth in the two groups of nude mice was evaluated (Fig. 7A). Tumor volume was measured weekly and a growth curve was plotted. The tumor volume of the SGC7901-pCDNA3.1-PR1 group was smaller compared with that of the SGC7901-pCDNA3.1 group, and the difference was statistically significant $(\mathrm{P}<0.05$; Fig. 7B).

IncRNARP1-163G9.1 inhibits tumor cell proliferation in vivo. Tumor tissue was isolated, the tumor volume and size were measured and representative tumor samples were subjected to hematoxylin and eosin staining to determine the nature of the tumor tissues (Fig. 7C and D). Statistical analysis of tumor volume and size demonstrated that there was a significant difference between the experimental and control
A

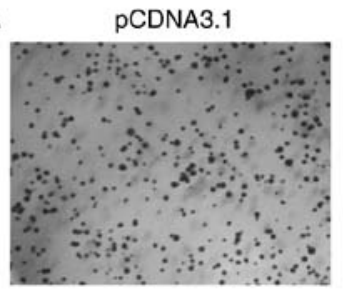

B PCDNA3.1-PR1

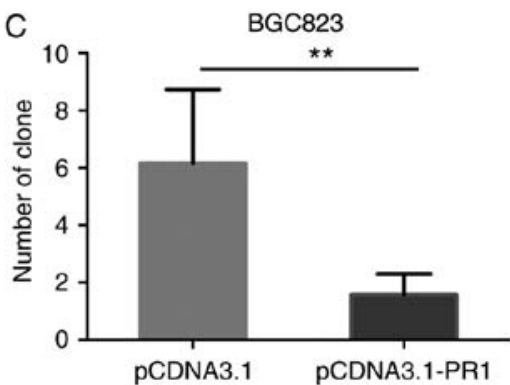

Figure 4. Cloning assay for detecting the effect of lncRNA RP1-163G9.1 on tumor cell clone formation. (A and B) Clone formation representative results for empty and overexpressed lncRNA RP1-163G9.1 cell strains of BGC823, respectively. (C) Statistical analysis of the data from the three independent experiments (mean \pm standard deviation). The clonogenic ability of pCDNA3.1 BGC823 cells was significantly higher compared with that in the pCDNA3.1-PR1 group $\left({ }^{* *} \mathrm{P}<0.01\right)$. IncRNA, long non-coding RNA.

groups $(\mathrm{P}<0.05$; Fig. 7E and $\mathrm{F})$. Therefore, these results revealed that lncRNARP1-163G9.1 overexpression can inhibit tumor growth in vivo.

\section{Discussion}

Based on the previous chip assay results, the present study was the first to report that the expression of IncRNA RP1-163G9.1 was downregulated in GA tissues. RT-qPCR results from $112 \mathrm{GA}$ tissues indicated that the rate of downregulation was $75.89 \%$ (85/112), and it was 23.94-fold lower in cancer samples compared with paired non-cancerous tissues. The present investigation further revealed that low expression of lncRNA RP1-163G9.1 was associated with increased invasiveness of GA, lymph node metastasis, larger tumor size and increased expression of the immunohistochemical marker Ki-67. Furthermore, the overexpression of lncRNA RP1-163G9.1 was shown to inhibit GA cell invasion and colony formation, cause a decrease of the number of cells in the $\mathrm{S}$ phase of the cycle in vitro, and reduce subcutaneous tumorigenesis in nude mice in vivo. These data support that lncRNA RP1-163G9.1 affects the proliferation ability of gastric cancer cells. In addition, the results suggested that the low expression of IncRNA RP1-163G9.1 in patients with GA is associated with tumor proliferative capacity.

Cancer is a complex disease, which was caused by multiple factors that are associated with changes in gene expression. Research mostly focuses on protein-coding genes. However, recently, emerging studies have indicated that lncRNAs are not only involved in the occurrence and development of human tumors (18), but may also be used as new type of biomarker and therapeutic target $(19,20)$. More evidence has demonstrated that IncRNAs are closely associated with human cancer, and play important roles in the regulation of cell proliferation 

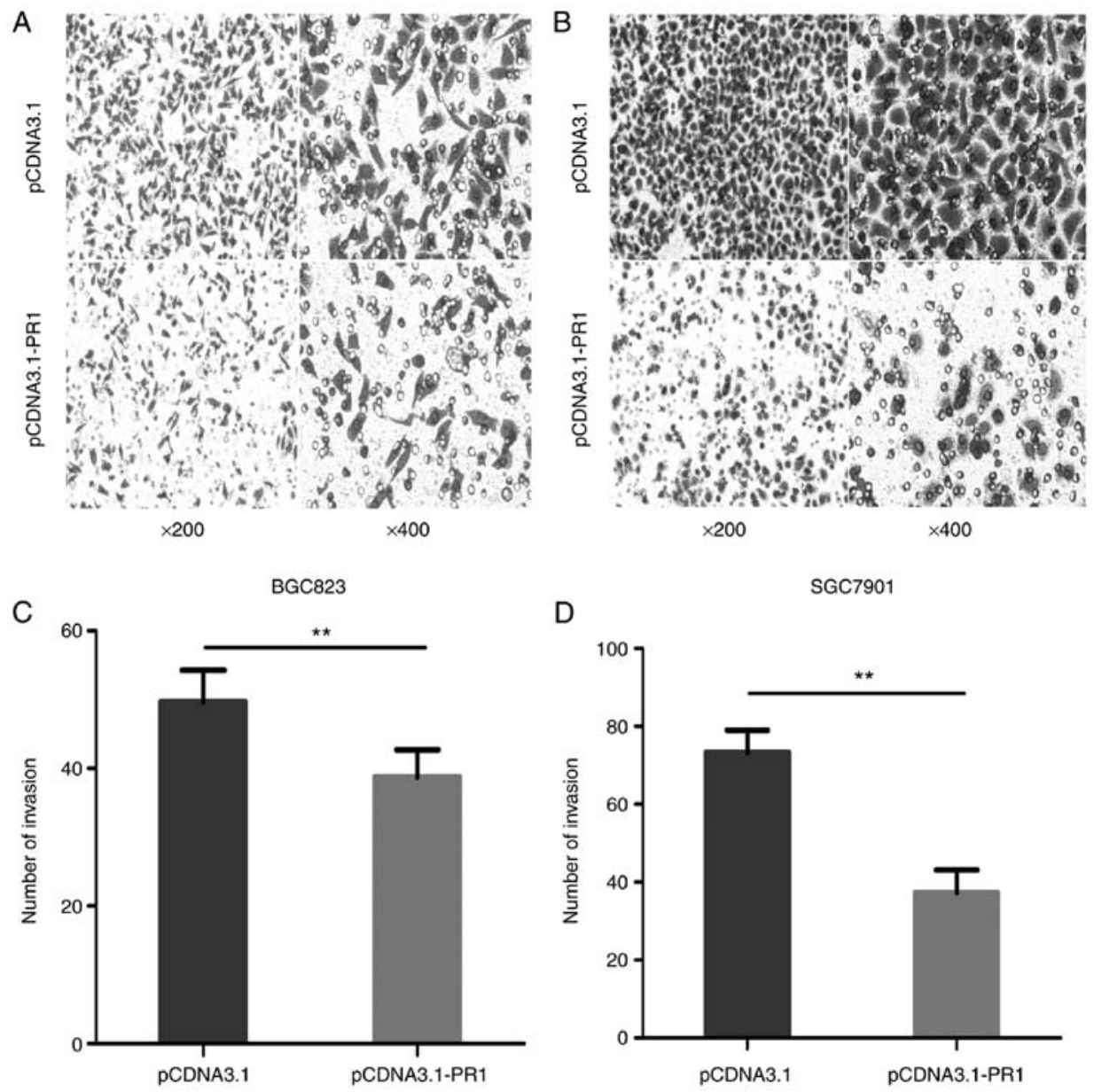

Figure 5. Transwell assay for detecting the effect of 1ncRNA RP1-163G9.1 on tumor cell invasion. Representative Transwell results for (A) BGC823 and (B) SGC7901 cells. Statistical analyses of (C) BGC823 and (D) SGC7901 cells. The data were obtained from three independent experiments (mean \pm standard deviation). ${ }^{* *} \mathrm{P}<0.01$. lncRNA, long non-coding RNA.

and apoptosis $(21,22)$, cell cycle, epigenetics and chromatin remodeling $(23,24)$, thereby participating in tumorigenesis and cancer progression. Therefore, lncRNAs are becoming an area of interest in research. IncRNAs are typically identified by the use of high-throughput chip technology. In addition, other methods, using bioinformatics techniques, have been used to predict the lncRNAs associated with tumors, including Improved Random Walk with Restart for IncRNA-Disease Association prediction (25) and KATZ measure for IncRNA-Disease Association prediction (26). These findings may be helpful in predicting gastric cancer-associated lncRNAs by building a new computer model.

In the present study, low expression of IncRNA RP1-163G9.1 was detected in GA tissues; however, its function in GA remained unclear. An overexpression vector of this lncRNA was therefore constructed and clones stably expressing lncRNA RP1-163G9.1 were screened. IncRNA RP1-163G9.1 exerted an effect on tumor proliferation and led to a cell cycle arrest in the $\mathrm{G}_{1}$ phase. The results of the present study were similar to the results obtained for other gastric cancer-associated-lncRNAs to some degree. For example, the lncRNA taurine-upregulated 1 was found to be overexpressed in GA through interacting with PRC2 to affect cell cycle progression (27). The present results further confirm that lncRNA dysregulation is closely associated with GA. Documented literature has indicated that the function of IncRNAs that participate in numerous signaling pathways is closely associated with their location in the cell (28). The results of our FISH assay revealed that 1ncRNA RP1-163G9.1 is located in the cytoplasm, and so it may be hypothesized that lncRNA RP1-163G9.1 may be associated with transcription regulation and protein translation.

Although the function of lncRNA RP1-163G9.1 was investigated in the present study, the underlying molecular mechanism requires further elucidation in future experiments. FISH demonstrated that lncRNA RP1-163G9.1 is located in the cytoplasm. According to current lncRNA research, it was hypothesized that lncRNA RP1-163G9.1 may act as a ceRNA that combines with endogenous miR and competes for the target gene (29). Alternatively, this lncRNA may act as a bait molecule for target gene recruitment $(30,31)$. As regards the molecular mechanisms involved in lncRNA function, it was previously demonstrated that lncRNAs may competitively combine with miRs, leading to various effects associated with oncogenesis, including cell proliferation, growth and metastasis (32). Thus, combined with bioinformatics technology, coding-non-coding gene co-expression (CNC) and ceRNA prediction analysis of lncRNA RP1-163G9.1 was performed in the present study. A total of 54 mRNAs with the same expression patterns were screened in CNC prediction (data not shown). Notably, the expression of $\sim 48$ of these was negatively correlated with lncRNA RP1-163G9.1 
A
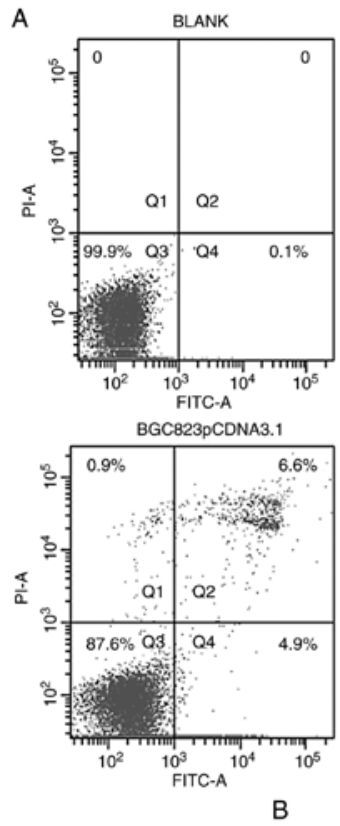

B
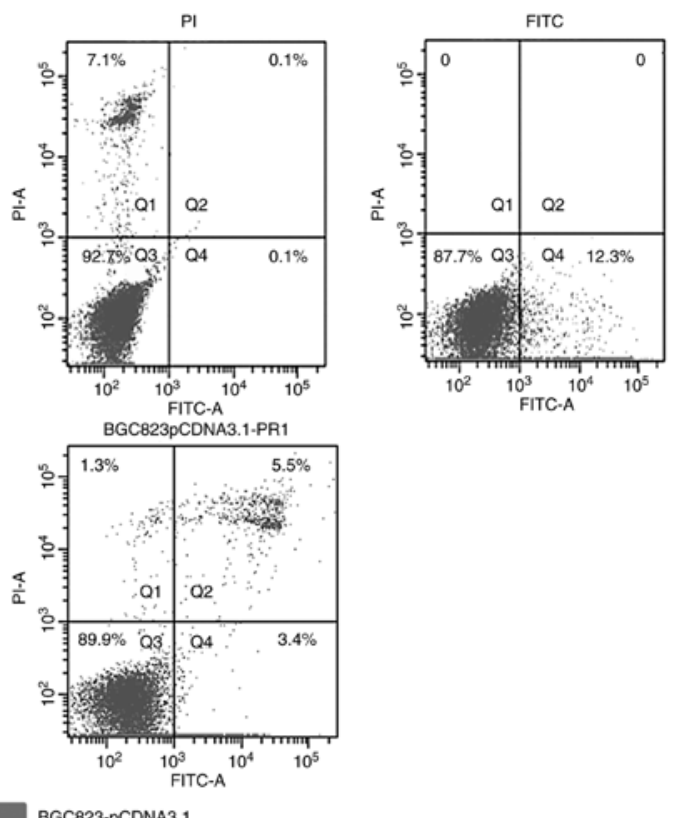

BGC-823PCDNA3.1-PR1

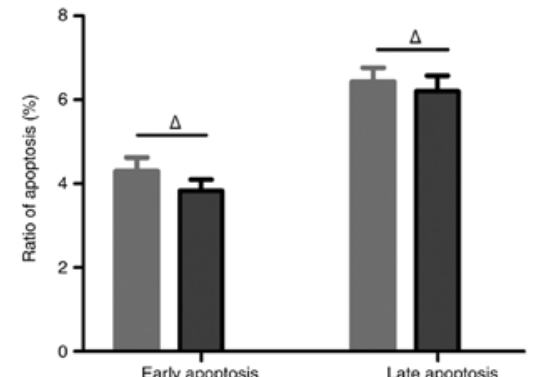

Early apoptosis

$\square$ Debris

\section{$\square$ Aggregates}

Dip G1

Dip G1: $55.99 \%$ at 105.95

Dip G2: $19.83 \%$ at 208.56

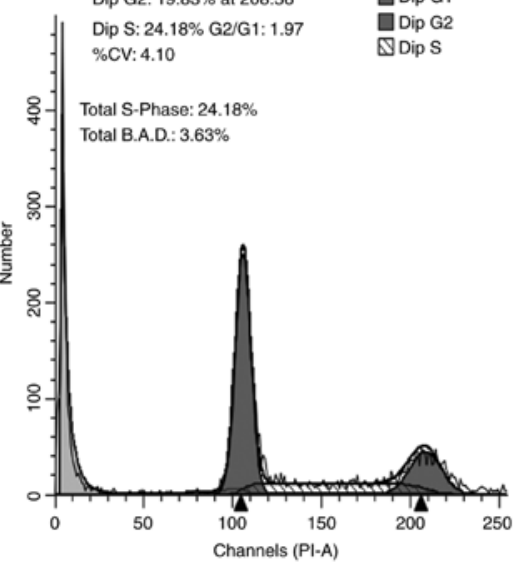

Dip S

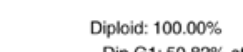

Dip G1: $50.82 \%$ at 113.87

Dip G2: $36.59 \%$ at 227.74

Dip S: $12.59 \%$ G2/G1: 2.00

Total S-Phase: $12.59 \%$

- Total B.A.D.: $8.59 \%$

్ㅡㅁ

ลิ-1

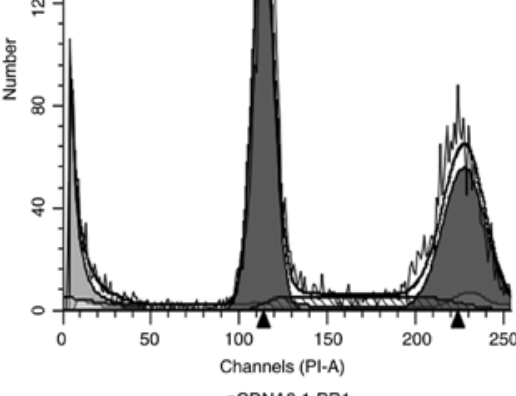

PCDNA3.1-PR1 $\square$ Debris

$\square$ Aggregates

Apoptosis

Dip G1

Dip G2
pCDNA3.1

BGC823

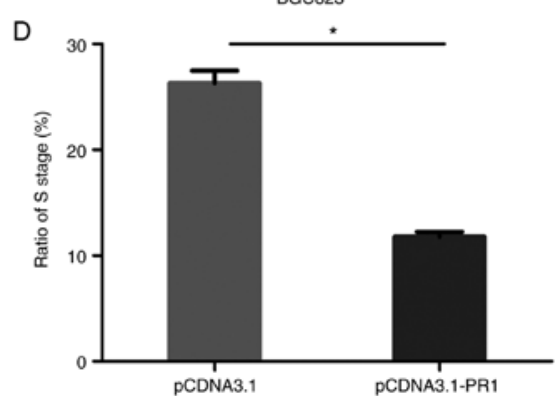

Figure 6. Analysis of cell apoptosis and cell cycle distribution in BGC823 cells stably overexpressing lncRNA RP1-163G9.1. (A) Representative images for apoptotic detection. (B) Statistical analysis revealed no statistically significant difference between the early apoptotic group $\left({ }^{\Delta} \mathrm{P}>0.05\right)$ and the late apoptotic group $\left({ }^{\Delta} \mathrm{P}>0.05\right)$. (C) Representative cell cycle images. (D) Statistical analysis. ${ }^{*} \mathrm{P}<0.05$. lncRNA, long non-coding RNA. 
A

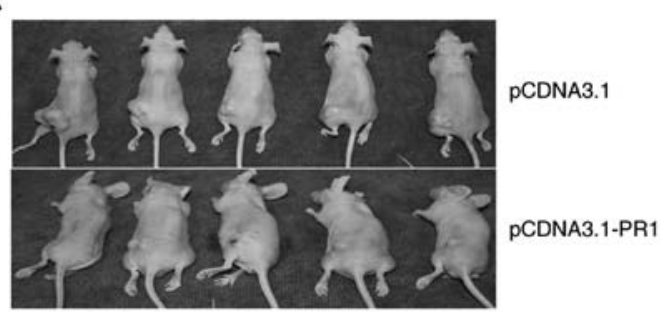

C
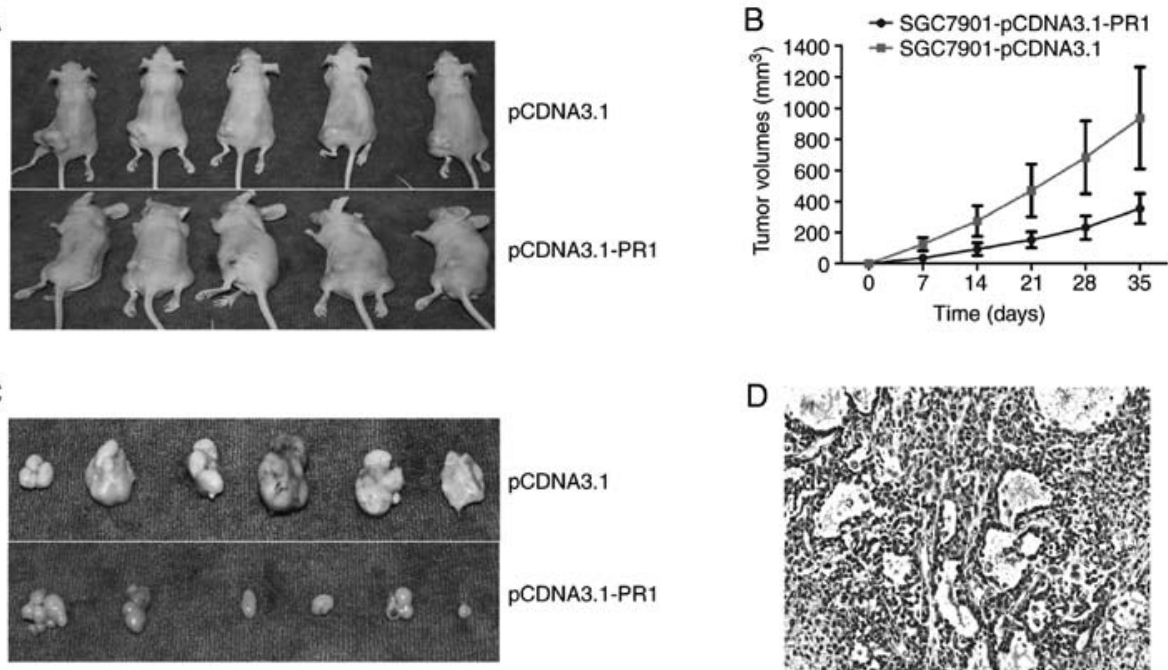

D

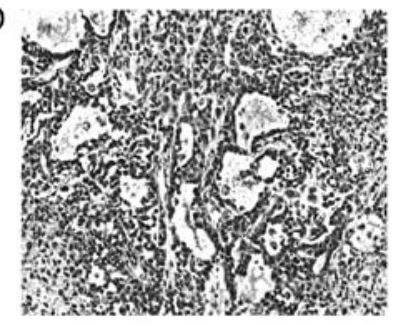

SGC7901

$\mathrm{E}$

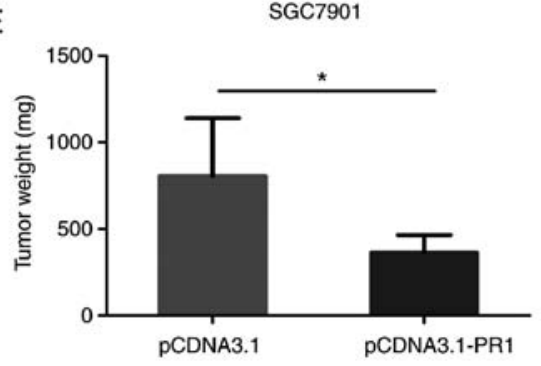

$\mathrm{F}$

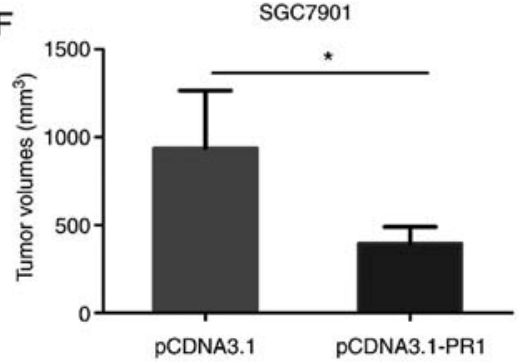

Figure 7. Subcutaneous tumorigenesis experiment using SGC7901 cells stably overexpressing lncRNA RP1-163G9.1. (A) Representative nude mice results are shown. (B) The growth curve revealed the difference was statistically significant $\left({ }^{*} \mathrm{P}<0.05\right)$. (C) Representative tumors. (D) Tumor hematoxylin and eosin staining. (E and F) Statistical analysis of tumor volume and tumor size from tumors obtained from two groups of nude mice. The results indicated that lncRNARP1-163G9.1 overexpression inhibited the proliferation of tumor cells in vivo. ${ }^{*} \mathrm{P}<0.05$.

and the expression of 6 was positively correlated. Furthermore, 41 miRs were predicted in ceRNA analysis (data not shown), which have nucleotide binding sites for lncRNA RP1-163G9.1. The results may be useful for further research on identifying the possible mechanism of IncRNA RP1-163G9.1 in GA. We hypothesized that lncRNA RP1-163G9.1 may not be related to apoptosis-associated gene expression or participate in cell apoptosis signaling pathway regulation. The exact role and molecular mechanism involved in the association of lncRNA RP1-163G9.1 with GA cell apoptosis requires further investigation.

There were certain limitations to the present study. These included an insufficient number of specimens and a lack of study of the underlying molecular mechanism. Next, a larger number of samples may be investigated to confirm its true clinical application, and the expression of lncRNA RP1-163G9.1 may be detected in collected blood samples (including whole blood, serum and plasma). If its expression is consistent with that in the tumor, a diagnostic cut-off value may be determined, which may represent a good diagnostic marker for gastric cancer. According to the results of bioinformatics prediction and the results of chip assay conducted on GA cells stably expressing lncRNA RP1-163G9.1, the high-score miRs and candidate target genes will be investigated to determine their association with the expression of lncRNA RP1-163G9.1. Furthermore, it was demonstrated that lncRNA RP1-163G9.1 is a proliferation index of gastric cancer and, based on this finding, the expression levels of genes that were related to cell proliferation will be detected at the protein and mRNA levels.
Taken together, the results of the present study indicate that 1ncRNA RP1-163G9.1 acts as an important regulator of tumor proliferation in GA and its expression is low in GA tissues. The findings of the present study suggest that lncRNA RP1-163G9.1 may be a potential target for GA diagnosis and therapy, and may provide a significant basis for understanding how lncRNAs are involved in proliferation regulation and tumor progression and treatment.

\section{Acknowledgements}

Not applicable.

\section{Funding}

The present study was supported by the Innovation Team Foundation of Fuzhou General Hospital (grant no. 2014CXTD04).

\section{Availability of data and materials}

All datasets generated and analyzed in the present study are available from the corresponding author on reasonable request.

\section{Authors' contributions}

BS was involved in the design of the study, was responsible for collecting the materials, conducting the literature review, 
performing the relevant experiments and writing the article. YD participated in the collection, preservation, quality control and RNA extraction of some specimens. FZ, KL, XO and $\mathrm{KW}$ were involved in the acquisition of specimens and the collection of the clinical data of the patients. FZ participated in the cell culture and animal tumor formation experiments. $\mathrm{KL}$ participated in the design of some experiments. KW was involved in the RNA extraction from some specimens and real-time RT-qPCR detection. XO participated in H\&E staining. QH was responsible for the design of the whole study and revision of the manuscript. All the authors have read and approved the final version of this manuscript.

\section{Ethics approval and consent to participate}

The present study was approved by the Fuzhou General Hospital Ethics Committee. All patients provided permission for the use of their tissue samples for research purposes.

\section{Patient consent for publication}

Not applicable.

\section{Competing interests}

The authors declare that they have no competing interests.

\section{References}

1. Wang K, Ruan J, Qian Q, Song H, Bao C, Zhang X, Kong Y, Zhang $\mathrm{C}$, Hu G, Ni J and Cui D: BRCAA1 monoclonal antibody conjugated fluorescent magnetic nanoparticles for in vivo targeted magnetofluorescent imaging of gastric cancer. J Nanobiotechnology 9: 23, 2011.

2. Wu WK, Lee CW, Cho CH, Cho CH, Fan D, Wu K, Yu J and Sung JJ: MicroRNA dysregulation in gastric cancer: A new player enters the game. Oncogene 29: 5761-5771, 2010.

3. Yang L: Incidence and mortality of gastric cancer in China. World J Gastroenterol 12: 17-20, 2006.

4. Nammi D, Srimath-Tirumala-Peddinti RC and Neelapu NR: Identification of drug targets in helicobacter pylori by in silico analysis: Possible therapeutic implications for gastric cancer. Curr Cancer Drug Targets 16: 79-98, 2016.

5. Sierzega M, Kaczor M, Kolodziejczyk P, Kulig J, Sanak M and Richter P: Evaluation of serum microRNA biomarkers for gastric cancer based on blood and tissue pools profiling: The importance of miR-21 and miR-331. Br J Cancer 117: 266-273, 2017.

6. Djebali S, Davis CA, Merkel A, Dobin A,Lassmann T, Mortazavi A, Tanzer A, Lagarde J, Lin W, Schlesinger F, et al: Landscape of transcription in human cells. Nature 489: 101-108, 2012.

7. Wiberg RA, Halligan DL, Ness RW, Necsulea A, Kaessmann H and Keightley PD: Assessing recent selection and functionality at long noncoding RNA loci in the Mouse genome. Genome Bio Evol 7: 2432-2444, 2015.

8. Chen X, Sun Y, Cai R, Wang G, Shu X and Pang W: Long noncoding RNA: Multiple players in gene expression. BMB Rep 51: 280-289, 2018

9. Terashima M, Tange S, Ishimura A and Suzuki T: MEG3 long noncoding RNA contributes to the epigenetic regulation of epithelial-mesenchymal transition in lung cancer cell lines. J Biol Chem 292: 82-99, 2017.

10. Ma B, Wang J, Song Y, Gao P, Sun J, Chen X, Yang Y and Wang Z: Upregulated long intergenic noncoding RNA KRT18P55 acts as a novel biomarker for the progression of intestinal-type gastric cancer. Onco Targets Ther 9: 445-453, 2016.

11. Liu XH, Sun M, Nie FQ, Ge YB, Zhang EB, Yin DD, Kong R, Xia R, Lu KH, Li JH, et al: LncRNA HOTAIR functions as a competing endogenous RNA to regulate HER2 expression by sponging miR-331-3p in gastric cancer. Mol Cancer 13: 92, 2014.
12. Sun Y, Pan J, Zhang N, Wei W, Yu S and Ai L: Knockdown of long non-coding RNA H19 inhibits multiple myeloma cell growth via NF-kappaB pathway. Sci Rep 7: 18079, 2017.

13. Zhang EB, Han L, Yin DD, Kong R, De W and Chen J: c-Mycinduced, long, noncoding $\mathrm{H} 19$ affects cell proliferation and predicts a poor prognosis in patients with gastric cancer. Med Oncol 31: 914, 2014.

14. Zhuang M, Gao W, Xu J, Wang P and Shu Y: The long non-coding RNA H19-derived miR-675 modulates human gastric cancer cell proliferation by targeting tumor suppressor RUNX1. Biochem Biophys Res Commun 448: 315-322, 2014.

15. Wu S, Zheng C, Chen S, Cai X, Shi Y, Lin B and Chen Y: Overexpression of long non-coding RNA HOTAIR predicts a poor prognosis in patients with acute myeloid leukemia. Oncol Lett 10: 2410-2414, 2015.

16. Dang Y, Lan F, Ouyang X, Wang K, Lin Y, Yu Y, Wang L, Wang Y and Huang Q: Expression and clinical significance of long non-coding RNA HNF1A-AS1 in human gastric cancer. World J Surg Oncol 13: 302, 2015.

17. Bai J, Yong HM, Chen FF, Mei PJ, Liu H, Li C, Pan ZQ, Wu YP and Zheng JN: Cullin1 is a novel marker of poor prognosis and a potential therapeutic target in human breast cancer. Ann Oncol 24: 2016-2022, 2013.

18. Yang B, Wei ZY, Wang BQ, Yang HC, Wang JY and Bu XY: Down-regulation of the long noncoding RNA-HOX transcript antisense intergenic RNA inhibits the occurrence and progression of glioma. J Cell Biochem 119: 2278-2287, 2018.

19. Tong YS, Wang XW, Zhou XL, Liu ZH, Yang TX, Shi WH, Xie HW, Lv J, Wu QQ and Cao XF: Identification of the long non-coding RNA POU $3 F 3$ in plasma as a novel biomarker for diagnosis of esophageal squamous cell carcinoma. Mol Cancer 14: 3, 2015.

20. Ozes AR, Wang Y, Zong X, Fang F, Pilrose J and Nephew KP: Therapeutic targeting using tumor specific peptides inhibits long non-coding RNA HOTAIR activity in ovarian and breast cancer. Sci Rep 7: 894, 2017.

21. Guo JQ, Li SJ and Guo GX: Long noncoding RNA AFAP1-AS1 promotes cell proliferation and apoptosis of gastric cancer cells via PTEN/p-AKT pathway. Dig Dis Sci 62: 2004-2010, 2017.

22. Liu G, Xiang T, Wu QF and Wang WX: Long noncoding RNA H19-derived miR-675 enhances proliferation and invasion via RUNX1 in gastric cancer cells. Oncol Res 23: 99-107, 2016.

23. Lee JJ, Kim M and Kim HP: Epigenetic regulation of long noncoding RNA UCA1 by SATB1 in breast cancer. BMB Rep 49: 578-583, 2016.

24. Liu Z, Chen Z, Fan R, Jiang B, Chen X, Chen Q, Nie F, Lu K and Sun M: Over-expressed long noncoding RNA HOXA11-AS promotes cell cycle progression and metastasis in gastric cancer. Mol Cancer 16: 82, 2017.

25. Chen X and Yan GY: Novel human lncRNA-disease association inference based on IncRNA expression profiles. Bioinformatics 29: 2617-2624, 2013

26. Chen X, Yan CC, Zhang X and You ZH: Long non-coding RNAs and complex diseases: From experimental results to computational models. Brief Bioinform 18: 558-576, 2017.

27. Zhang E, He X, Yin D, Han L, Qiu M, Xu T, Xia R, Xu L, Yin R and De W: Increased expression of long noncoding RNA TUG1 predicts a poor prognosis of gastric cancer and regulates cell proliferation by epigenetically silencing of p57. Cell Death Dis 7: e2109, 2016.

28. Atanasovska B, Rensen SS, van der Sijde MR, Marsman G, Kumar V, Jonkers I, Withoff S, Shiri-Sverdlov R, Greve JWM, Faber KN, et al: A liver-specific long noncoding RNA with a role in cell viability is elevated in human nonalcoholic steatohepatitis. Hepatology 66: 794-808, 2017.

29. Xia T, Liao Q, Jiang X, Shao Y, Xiao B, Xi Y and Guo J: Long noncoding RNA associated-competing endogenous RNAs in gastric cancer. Sci Rep 4: 6088, 2014.

30. Hu Y, Wang J, Qian J, Kong X, Tang J, Wang Y, Chen H, Hong J, Zou W, Chen Y, et al: Long noncoding RNA GAPLINC regulates CD44-dependent cell invasiveness and associates with poor prognosis of gastric cancer. Cancer Res 74: 6890-6902, 2014.

31. He H, Wang N, Yi X, Tang C and Wang D: Long non-coding RNA H19 regulates E2F1 expression by competitively sponging endogenous miR-29a-3p in clear cell renal cell carcinoma. Cell Biosci 7: 65, 2017.

32. Wang H, Yu Y, Fan S and Luo L: Knockdown of long noncoding RNA TUG1 inhibits the proliferation and cellular invasion of osteosarcoma cells by sponging miR-153. Oncol Res 26: 665-673, 2018 